\title{
Complex and Coherent Risk Measure
}

\author{
M.C. MIGLIONICO, G. D'ANGELO
}

Department of Architecture and Industrial design "Luigi Vanvitelli" Second University of Naples (SUN), Aversa, Italy,

ABSTRACT: Coherent risk measures have been introduced by Artzner et al. in financial markets where the set of states $\Omega$ is finite. This theory has been extended by Delbaen to cases where $\Omega$ is infinite. The financial markets are constantly evolving and the classical definition of risk measures is "static". Hence, the need to study its complexity and evolution. "Complexity theory" focuses on the interdisciplinary study of its systems. In its evolution, the study of complex adaptive systems and the emergent phenomena associated with them are of fundamental importance. In this paper, we consider the coherent risk measure and their interpretation as complex adaptive system.

KEYWORD: Coherent risk measure; complex adaptive system.

\section{COHERENT RISK MEASURE}

Although several papers (including an earlier version of this one) define risk in terms of changes in values between two dates, we argue that because risk is related to the variability of the future value of a position, due to market changes or more generally to uncertain events, it is better to consider only future values.

A first crude, but crucial, measurement of the risk of a position will be whether its future value belongs or does not belong to the subset of acceptable risks, as decided by a supervisor such as:

a) a regulator who takes into account the unfavourable states when allowing a risky position that may draw on the resources of the government - for example as a guarantor of last resort;

b) an exchange's clearing firm, which has to make good on the promises to all parties of transactions being securely completed;

c) an investment manager who knows that his firm has basically given its traders an exit option in which the strike "price" consists of being fired in the event of big trading losses on one's position.

Let $\mathrm{G}$ be the set of all risks, that is the set of all real-valued functions on $\Omega$. Since $\Omega$ is assumed to be finite, $\mathrm{G}$ can be identified with $\mathrm{Rn}$, where $\mathrm{n}=\operatorname{card}(\Omega)$. The cone of nonnegative elements in $\mathrm{G}$ shall be denoted by (L+), its negative by (L-).

We call "risk measure" the number $\rho(\mathrm{X})$ assigned by the measure $\rho$ to the risk $X$ will be interpreted as the minimum extra cash the agent has to add to the risk position X, and invest "prudently," that is in the reference instrument, to be allowed to proceed with his plans. If it is negative, the cash amount $-\rho(X)$ can be withdrawn from the position or it can be received as restitution, as in the case of organized markets for financial futures.

A risk measure satisfying the following four axioms of translation invariance, subadditivity, positive homogeneity and monotonicity is called coherent.

$\checkmark$ Translation invariance: Let $r$ a strictly positive price (or total return) in any state of nature at date $T$, for all $X \in G$ and all real numbers $\alpha$, we have

$$
\rho(X+\alpha \cdot r)=\rho(X)-\alpha
$$

Subadditivity: For all $\mathrm{X} 1$ and $\mathrm{X} 2 \in \mathrm{G}$, $\rho\left(X \_1+X \_2\right) \leq \rho\left(X \_1\right)+\rho\left(X \_2\right)$

We contend that this property, which could be stated in the brisk form "a merger does not create extra risk," is a natural requirement:

a) If an exchange's risk measure were to fail to satisfy this property, then, for example, an individual wishing to take the risk $X_{1}+X_{2}$ may open two accounts, one for the risk $X_{1}$ and the other for the risk $X_{2}$, incurring the smaller margin requirement of $\rho\left(X_{1}\right)+\rho\left(X_{2}\right)$, a matter of concern for the exchange.

b) If a firm were forced to meet a requirement of extra capital which did not satisfy this property, the firm might be motivated to break up into two 
separately incorporated affiliates, a matter of concern for the regulator.

c) Bankruptcy risk inclines the firm to require less capital from a group without "fire- walls" between various business units than it does require when one "unit" is protected from liability attached to the failure of another "unit."

d) Supposes that two desks in a firm compute in a decentralized way the measures $\rho\left(X_{1}\right)$ and $\rho\left(X_{2}\right)$ of the risks they have taken. If the function $\rho$ is subadditive, the supervisor of the two desks can count on the fact that $\rho\left(X_{1}\right)+\rho\left(X_{2}\right)$ is a feasible guarantee relative to the global risk $X_{1}+X_{2}$. If he has an amount $\mathrm{m}$ of cash available for their joint business, he knows that imposing limits $\mathrm{m}_{1}$ and $\mathrm{m}_{2}$ with $\mathrm{m}=\mathrm{m}_{1}+\mathrm{m}_{2}$, allows him to decentralize his cash constraint into two cash constraints, one per desk. Similarly, the firm can allocate its capital among managers.

$\checkmark$ Positive homogeneity: For all $\lambda \geq 0$ and for all $X \in G, \rho(\lambda X)=\lambda \rho(X)$

$\checkmark$ Monotonicity: For all $\mathrm{X}$ and $\mathrm{Y} \in \mathrm{G}$ with $\mathrm{X} \leq$ Y; we have $\rho(Y) \leq \rho(X)$

\section{COMPLEX ADAPTIVE SYSTEMS}

In physics, a complex system is a system in which the individual parts are affected by local interactions, short-range, which cause changes in the overall structure

The characteristics of complex systems are:

1) Many components more or less complex: in the more complex systems, subsystems (i.e. components) are themselves high complexity; components can be "hardware" (molecules, physical processors, cells, individuals) or "software" processing units (virtual);

2) Interactions between components: components interact by passing information (in the form of energy, matter, or digital information); the amount of connections and the presence of substructures and recursive feedback loops (socalled "rings") increase the complexity of the system, but the information that the components are exchanged may not be too many (otherwise the system becomes chaotic ) nor too little (the system will "crystallize");

3) Absence of a "pyramid" hierarchy: if there is a single component, which alone governs the behaviour of all, the system cannot be complex; its description, in fact, can easily be reduced to that of the subsystem - leader; different is the case of complex systems "hologram", in which each component has information about the system as a whole (for example, each cell contains all the genetic information of the organism to which it belongs).
These are supplemented by a fourth defining characteristic of complex adaptive systems:

4) adaptive interaction with the environment: the system is much more complex, as there are many factors that affect its adaptation to the environment (which must take into account the model): incidence of random factors, learning, interaction with the observer of the system, and so on; while the system evolves, its subsystems co-evolve by developing strategies for coadaptation (symbiosis, cooperation, communication, etc.).

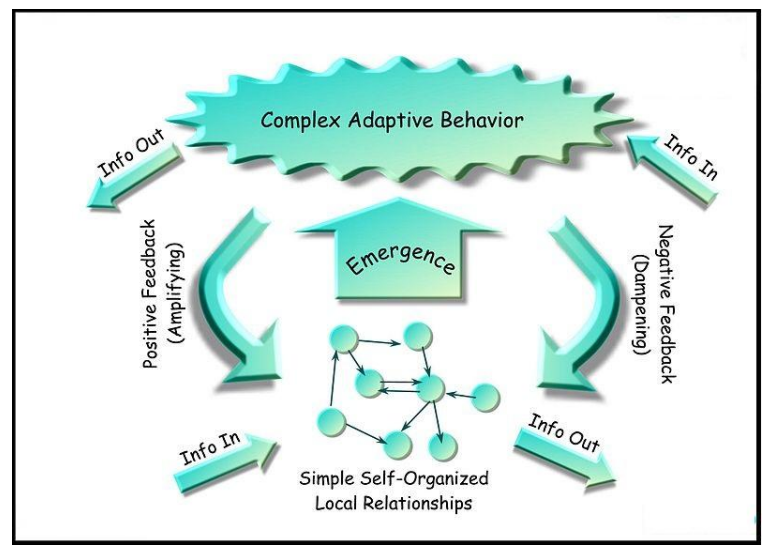

Figure 1. Complex adaptive system

Complexity theory studies the interdisciplinary nature of complex adaptive systems and emergent phenomena associated with them.

Many natural systems (e.g., brains, immune systems, ecologies, societies) and increasingly, many artificial systems (parallel and distributed computing systems, artificial intelligence systems, artificial neural networks, evolutionary programs) are characterized by apparently complex behaviours that emerge as a result of often nonlinear spatialtemporal interactions among a large number of component systems at different levels of organization. These systems have recently become known as Complex Adaptive Systems.

The system $\mathrm{G}$ (set of all the risks) is characterized by the fact that it is not possible to establish the laws that govern it and is therefore unpredictable. Its behaviour is not linear and can, in certain cases, absorb large disruptions without having major effects while on the contrary, certain small perturbations can cause catastrophic effects. The "system" G, having no general law that governs it, is unpredictable, however, it can be characterized by typical behaviours through which it is for example possible to predict the appearance of a phenomenon but not in general a precise time in which this can take place. The system $G$ is a complex adaptive system.

Different groups of traders have different expectations about future prices and future dividends. For example, one group might be 
fundamentalists, believing that asset prices return to their fundamental equilibrium price, whereas another group might be chartists, extrapolating patterns in past prices. Traders choose their trading strategy according to an evolutionary 'fitness measure', such as accumulated past profits. Agents are boundly rational, in the sense that most traders choose strategies with a higher fitness.

\section{COHERENT RISK MEASURE AS COMPLEX ADAPTIVE SYSTEM}

We consider $\lambda$ function of the interaction between the components of

$$
\lambda\left(X_{1}, X_{2}, \ldots, X_{n}\right)
$$

with

$$
X_{1}, X_{2}, \ldots, X_{n} \in G
$$

and $\alpha$ function of the characteristics of the external environment that may lead an individual to invest an amount of money $\alpha$, the risk measure $\rho^{*}(\mathrm{X})$ varying the parameters $\alpha$ and $\lambda$ is a coherent risk measure, it enjoys all the axioms of coherent risk measures:

Positive homogeneity:

$$
\rho^{*}\left[\lambda\left(X_{1}, X_{2}, \ldots, X_{n}\right) \cdot X\right]=\lambda \rho^{*}(X)
$$

Subadditivity: For all $\mathrm{X}_{1}$ and $\mathrm{X}_{2} \in \mathrm{G}$, $\rho^{*}\left(X_{1}+X_{2}\right) \leq \rho^{*}\left(X_{1}\right)+\rho^{*}\left(X_{2}\right)$
Translation invariance: For all $\mathrm{X} \in \mathrm{G}$ and all real numbers $\alpha\left(\alpha_{1}, \alpha_{2}, \ldots, \alpha_{\mathrm{n}}\right)$ we have

$$
\rho^{*}\left(X+\alpha\left(\alpha_{1}, \alpha_{2}, \ldots, \alpha_{m}\right) r\right)=\rho^{*}(X)-\alpha
$$

Monotonicity: For all $\mathrm{X}$ and $\mathrm{Y} \in \mathrm{G}$ with $\mathrm{X} \leq \mathrm{Y}$; we have $\rho^{*}(\mathrm{Y}) \leq \rho^{*}(\mathrm{X})$

The variables $\alpha$ and $\lambda$ are stochastic variables that represent the probability of investors to invest funds.

The set of all coherent risk measures defined above:

$\left\{\rho_{i}^{*}(X)\right\}$ is also a complex adaptive system, consisting of several components, which are also complex. There is the interaction between the parts due to the variability of $\lambda$, the non-linear financial markets ensures that there is a pyramidal hierarchy and finally, the interaction is ensured and the adaptation to the external environment guaranteed by the variability of $\alpha$.

\section{REFERENCES}

[1] P. Artzner, F. Delbaen, J.M. Eber, D. Heath (1999) Coherent measure of risk;

[2] G. D’Angelo (2014) Complexity and landscape: interactions and analogies

[3] C.H. Hommes (2001) Financial markets as nonlinear adaptive evolutionary systems

[4] M.C. Miglionico F. Parillo, (2012) An Application in Bank Credit Risk Management System employing a BP Neural Network based on sfloat24 custom math library using a low cost FPGA device 